\title{
Rectus sheath hematoma: a potentially life threatening complication following caesarean delivery
}

\author{
Sonal Dube ${ }^{1 *}$, Meenakshi Dube ${ }^{2}$, Saumitra Dube ${ }^{3}$
}

\begin{abstract}
${ }^{1}$ Department of Obstetrics and Gynecology, Pt. J. N. M. Medical College, Raipur, Chhattisgarh, India ${ }^{2}$ Department of Obstetrics and Gynecology, Shri Ram Hospital, Raipur, Chhattisgarh, India

${ }^{3}$ Department of Surgery, SRM Medical College, Chennai, Tamil Nadu, India
\end{abstract}

Received: 17 July 2018

Accepted: 01 September 2018

\author{
*Correspondence: \\ Dr. Sonal Dube, \\ E-mail: sonaldube06@gmail.com
}

Copyright: (c) the author(s), publisher and licensee Medip Academy. This is an open-access article distributed under the terms of the Creative Commons Attribution Non-Commercial License, which permits unrestricted non-commercial use, distribution, and reproduction in any medium, provided the original work is properly cited.

\begin{abstract}
Rectus sheath hematoma (RSH) is a rare but potentially life threatening complication of caesarean delivery. The nonspecific nature of entity, lower incidence of disorder and acute presentation may posses' difficulty in timely recognizing this. Present patient presented as acute abdominal pain 38 hours post caesarean section. This is an attempt to increase the awareness of this rare but potentially grave condition, as timely diagnosis and fast intervention saved present patient.
\end{abstract}

Keywords: Carnett's sign, Rectus sheath hematoma, Turner's sign

\section{INTRODUCTION}

Hematoma of rectus muscle is an acute or chronic collection of blood lying within the muscle or between the muscle and sheath. It is the most common primary non-neoplastic condition of rectus muscle and sheath. ${ }^{1}$ It may result from damage to inferior and superior epigastric arteries or from direct trauma to rectus muscle.

Rectus sheath is formed by aponeurosis of lateral abdominal muscles. Below the arcuate line of douglas, which is between umbilicus and pubis, all the aponeurosis pass in front of the rectus. Consequently, the posterior surface of the muscle under the arcuate line is covered only by relatively weak transversalis fascia and the peritoneum. Three to four tendinous insertions attach the rectus muscles to the enveloping fascia above arcuate line. These insertions contain multiple perforators from epigastric vessels supplying the overlying fascia and soft tissues. The perforators are most dense in periumbilical region. $^{2}$
As the muscle contracts, its length changes and the vessels must slide with it to avoid tearing. In case of inferior epigastric artery, the combination of its loose attachment with its stable and fixed perforating branches makes it prone to injury, during strong muscle contraction. This explains why $\mathrm{RSH}$ is more common in lower abdomen. ${ }^{3}$

Table 1: Causes of RSH.

Causes
Coughing and sneezing
Direct trauma and physical exertion
Pregnancy
Hypertension
Increasing age
Anticoagulant therapy
Amniocentesis, paracentesis, abdominal surgeries,
subcutaneous insulin injection
Bleeding diasthesis


Other causes of RSH are listed in Table 1 and possible surgical causes are summarized in Table 2. Clinical presentation may range from self-limiting condition with mild induration to hypovolemic shock, with associated morbidity and mortality.

Table 2: Possible surgical causes of RSH.

\begin{tabular}{|l|}
\hline Causes \\
\hline Vigorous retraction \\
\hline Inadequate haemostasis \\
\hline Needle laceration \\
\hline Slipped ligature \\
\hline Impaired mobility of muscles \\
\hline Sawing effect of abdominal wall sutures \\
\hline Excessive separation of muscle \\
\hline Excessive undermining of muscle \\
\hline
\end{tabular}

\section{CASE REPORT}

23-year-old female, primigravida with twin gestation underwent caesarean section for discordant twins. Her intra-operative period was uneventful. Immediate post operatively there was rise in blood pressure, so she was started nifedipine and her blood pressure was under control. Oral intake of fluids and semi solid food was started, and she was comfortable. Thirty-eight hours later she complained of acute pain in lower abdomen, assuming it to be the post-operative incision pain added dose of analgesia was given and she was haemodynamically stable. Pain was partially relieved. Half an hour later she again complained of intense pain in lower abdomen so much so that she was unable to sleep.

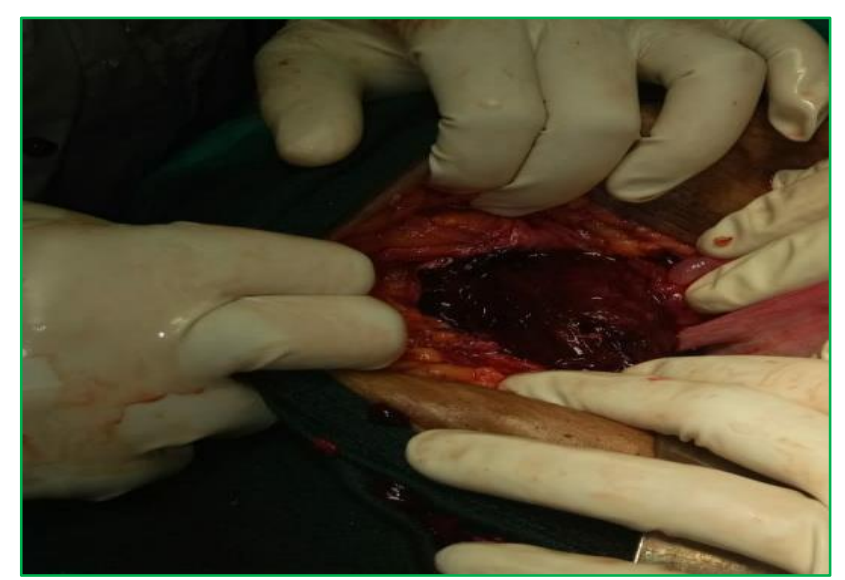

Figure 1: Hematoma in rectus muscle.

On examination there was tenderness $3 \mathrm{~cm}$ above incision line more so on left side. Her pulse was settled but her blood pressure was 90/60 mm hg. Keeping in mind her previous blood pressure values, this change was definitely alarming. Her abdomen had soft distension, but good bowel sounds were present. Uterus was well contracted and there was no bleeding per vaginum. Per vaginum examination was within normal limits. Despite adequate fluid resuscitation her blood pressure remained low even reaching a value of 60/40 $\mathrm{mm}$ hg and eventually she had tachycardia. Clinically the possibility of surgical site bleed was strong, but no fluid collection was appreciated clinically and Carnett's sign could not be elicitated due to extreme pain. Also, there was fall in hemoglobin levels from $12.5 \mathrm{~g} / \mathrm{dl}$ pre-operatively to $8.6 \mathrm{~g} / \mathrm{dl}$. There was mild leukocytosis (TLC-16,500) and fall in platelet counts (1.8 lac pre-operative to $1.2 \mathrm{lac}$ ). Her coagulation screen (BT/CT, PT and a PTT) was normal. Ultrasonography revealed a large heterogenous space occupying lesion anterior to uterus likely to represent hematoma of $20 \mathrm{~cm} * 18 \mathrm{~cm} * 7 \mathrm{~cm}$ and large amount of free fluid was seen in peritoneal cavity. She was immediately shifted for laprotomy. Two-pint blood and 2 FFP were transfused.

On opening the abdomen, about 4 fistful clots were removed below the rectus sheath (Figure 1). Bleeding point was seen in the transverse abdominalis muscle, about 2-3 cm lateral to margin of rectus muscle on left side. Bleeder was tied, and hemostasis was achieved. Incision line on uterus was intact with no bleeding, however about $300 \mathrm{ml}$ of dark coloured blood was washed from peritoneal cavity possibly from percolation from rectus muscle and peritoneum was bruised (Figure 2). Hematoma had crossed midline which caused diffuse tenderness of abdomen and hence, no specific lump was delineate. Two more pint of blood was transfused postoperatively. Her recovery was fast and satisfactory.

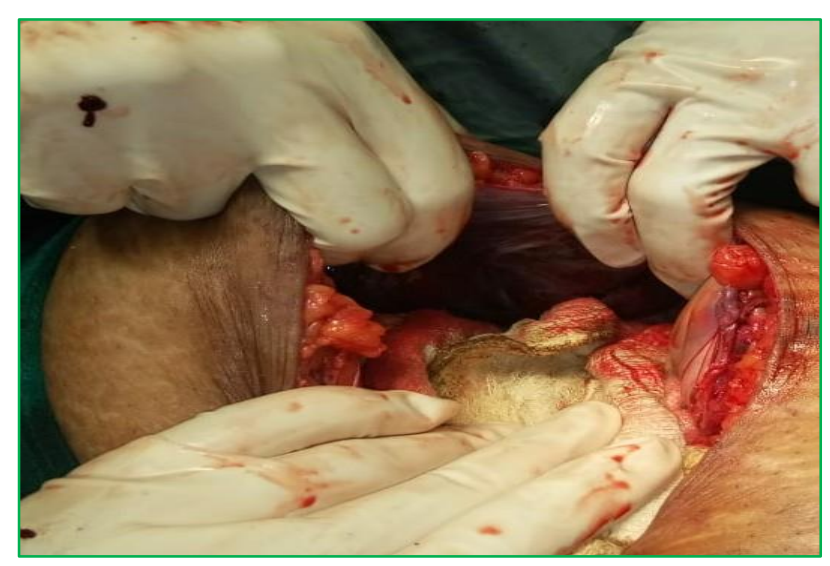

Figure 2: Intra-fascial extension of hematoma, causing bruising of peritoneum.

\section{DISCUSSION}

Rectus sheath hematoma is rare, although it has been known since the time of Hippocrates, Galen and Leonardo da vinci. ${ }^{2}$ It is $2-3$ times more common in females than males due to low muscle mass. ${ }^{4}$ It presents as painful, tender abdominal swelling of sudden onset. ${ }^{5}$ Present patient presented in similar manner. Although usually a benign self-limiting condition, RSH may be fatal, overall mortality rate is reported to be $4 \%{ }^{2,3}$ Most common symptoms include-abdominal pain (84-97\%), 
palpable abdominal wall mass (63-92\%) and tenderness (71\%). Other less common symptoms include- nausea and vomiting, tachycardia, orthostatic symptoms, hypotension, ecchymosis, syncope, peritoneal signs and fever. ${ }^{6,3}$ Pain could be of acute onset or develop over hours. Pain is usually described as severe, sharp, persistent and non-radiating. It is worse with movement and is associated with palpable abdominal mass. Generally, the abdominal mass in RSH does not cross the midline and, in contrast to an intraperitoneal mass, it remains conspicuous on tensing the abdominal wall musculature by head or leg raising. This is referred to as Fothergill's sign. ${ }^{7}$ Similarly, tenderness remains the same or increases with head raising and is referred to as Carnett's sign. Ecchymosis can be noted in the flanks or periumbilical areas, especially late in the course, and are referred to as Gray Turner's and Cullen's sign respectively. Ultrasonography is an inexpensive, safe and easy to use diagnostic method and is used as first line test in acute abdominal pain. ${ }^{8}$ It can be used as first line modality for diagnosis as well as monitoring. Typically, RSH appears on ultrasonography as spindle shaped in longitudinal scan and as ovoid mass on transverse and coronal section. It appears homogenous and sonolucent, but in the presence of clot could appear heterogenous. ${ }^{3}$ However USG has sensitivity of $80-90 \%$. CT scan is reported to have a sensitivity and specificity reaching about $100 \%$. Besides, it also provides information regarding size, location, origin, extent, location and nature of hematoma, and helps us exclude concomitant intra-abdominal pathologies. RSH typically appears as hyperdense mass, located posterior to the rectus abdominis muscle, with ipsilateral enlargement of muscle. Chronic hematoma may be iso/hypodense. On the basis of CT scan RSH may be classified as following:

Type-I: Unilateral, intramuscular, does not dissect fascial plane, no haemodynamic instability.

Type-II: Bilateral, some dissection between the muscle and transversalis fascia, no extension to pre-vesicle space, minimal or no haemodynamic instability.

Type-III: Most severe, Bilateral, large, dissects between the transversalis fascia and muscle into the peritoneum and prevesical space, haemodynamic instability is present. May require immediate surgical intervention.

Cost may be a limitation in resource poor setting. Role of MRI is limited only if the hematoma is older than 5 days and CT findings are inconclusive. In older cases it appears as a high intensity area, both on T1 and T2 weighted images. Management depends on the type of $\mathrm{RSH}$, associated haemodynamic instability and clinical presentation. Conservative management with rest, analgesia, antibiotics and cold compression is useful in milder case. Large hematomas causing haemodynamic instability, expanding haematomas and infected ones need surgical management. Evacuation followed by ligation of bleeding vessel and drainage, if necessary, is usually enough to prevent further complications. Other modalities may include transcatheter embolization technique with thrombin, gelform or coil which is alternative to surgery for conditions not responding to conservative management. Despite good surgical hemostasis RSH can occur even with trivial pathology like cough. In present case, although a CT scan was not done due to critical condition of patient, intra-operative finding revealed type III RSH. Close monitoring of patient and timely surgical intervention was a life saving measure.

\section{CONCLUSION}

$\mathrm{RSH}$ is reported to be top three causes of re-laparotomy post C-section along with PPH and intra-peritoneal bleed in literature. Medical training adequately emphasizes the recognition of common surgical intra-abdominal emergencies, however there is relative lack of emphasis and hence awareness about abdominal wall problems presenting as acute abdomen. Moreover, RSH is a great masquerader and can mimic other intra-abdominal pathologies, especially intra-peritoneal surgical leak as in the case of caesarean section. Hence, there is tendency of delayed diagnosis. High index of suspicion and appropriate intervention can save the life in massive RSH.

\section{Funding: No funding sources \\ Conflict of interest: None declared \\ Ethical approval: Not required}

\section{REFERENCES}

1. Joshi NR, Upadhyaya I. Post-caesarean Rectus Sheath Haematoma. Nepal J Obstet Gynaecol. 2015;10(2).

2. Awe JA, Soliman AM. Rectus sheath hematoma of the abdomen an uncommon diagnostic challenge. Glo Adv Res J Microbiol. 2013;2(9):159-63.

3. Hatjipetrou A, Anyfantakis D, Kastanakis M. Rectus sheath hematoma: a review of the literature. Int J Surg. 2015;13:267-71.

4. Dwarakanath L, Shobha UN, Jamuna R, Hema KR, Sowmya G. Rectus sheath hematoma-an uncommon complication following caesarean delivery. J Int Med Dent. 2014;1(1):32-4.

5. Wahi I. Post-caesarean rectus sheath hematoma: a case report. Marmara Med J. 2009:22(3):240-2.

6. Alla VM, Karman SM, Kaushik M, Porter J. Spontaneous rectus sheath hematoma. Western J Emerg Med. 2010;11(1):76.

7. Osinbowale O, Bartholomew JR. Rectus sheath hematoma. Vasc Med. 2008;13:275-9.

8. Stoker J, van Randen A, Laméris W, Boermeester MA. Imaging patients with acute abdominal pain. Radiology. 2009;253(1):31-46.

Cite this article as: Dube S, Dube M, Dube S. Rectus sheath hematoma: a potentially life threatening complication following caesarean delivery. Int J Reprod Contracept Obstet Gynecol 2018;7:4289-91. 